\title{
A Robot Assisted High-flow Portable Cyclone Sampler for Bacterial and SARS-CoV-2 Aerosols
}

Aerosol and Air Quality Research

\section{OPEN ACCESS}

Received: June 7, 2021

Revised: September 9, 2021

Accepted: November 18, 2021

${ }^{*}$ Corresponding Author:
yao@pku.edu.cn

Publisher:

Taiwan Association for Aerosol Research

ISSN: $1680-8584$ print

ISSN: 2071-1409 online

Copyright: The Author(s). This is an open access article distributed under the terms of the Creative Commons Attribution License (CC BY 4.0), which permits unrestricted use, distribution, and reproduction in any medium, provided the original author and source are cited.

\author{
Xinyue $\mathrm{Li}^{1}$, Haoxuan Chen ${ }^{1}$, Xiao $\mathrm{Qi}^{2}$, Yijiao Peng ${ }^{1}$, Lian Zhou ${ }^{3}$, Jianxin $\mathrm{Ma}^{2}$, \\ Maosheng $\mathrm{Yao}^{{ }^{*}}$
}

${ }^{1}$ State Key Joint Laboratory of Environmental Simulation and Pollution Control, College of Environmental Sciences and Engineering, Peking University, Beijing 100871, China

${ }^{2}$ Center for Disease Control and Prevention of Chaoyang District of Beijing, Beijing, China

${ }^{3}$ Department of Environment and Health, Jiangsu Provincial Center for Disease Control and Prevention, Nanjing, China

\section{ABSTRACT}

Airborne transmission of infectious diseases attracts great attention since the COVID-19 pandemic. Yet, there has been an intense dispute about aerosol transmission of the disease, which is largely due to lack of qualified instruments for studying the subject. Air sampling plays a critical role in all air pollution related study, and particularly critical for airborne pathogen detection. Here, we designed and evaluated a portable and high volume ( $400 \mathrm{~L} \mathrm{~min}^{-1}$ ) cyclone sampler named as Yao-CSpler using aerosolized Polystyrene (PS) uniform microspheres, Bacillus subtilis var. niger, Pseudomonas fluorescens, and both indoor and outdoor air particles. The experimental cutoff size of the Yao-CSpler was demonstrated to be $0.58 \mu \mathrm{m}$ (while the calculated theoretical value is $1.84 \mu \mathrm{m}$ ), and the sampler has shown stable microbial collection performances for bacteria, fungi, and even viruses. The sampler had a physical collection efficiency of close $100 \%$ for particles of larger than $1 \mu \mathrm{m}$. Jet-to-liquid distance and sampling duration were shown to substantially influence the sampler performance. Given the same sampling duration, the performances of the Yao-CSpler were significantly higher than those of the traditional BioSampler (SKC Inc.) in terms of samples' bacterial diversity. The developed sampler coupled with a robot has been successfully applied to sampling airborne SARS-CoV-2 in both Wuhan and Beijing during the COVID-19 outbreaks. With a high sampling flow, the Yao-CSpler was shown to be able to collect the SARS-CoV-2 with a detectable concentration level down to 9-219 viruse $\mathrm{m}^{-3}$ in clinical settings housing COVID-19 patients. Further more efficient bioaerosol sampler, which is able to rapidly capture low level pathogenic agents, is urgently required to better understand and confront airborne transmission of infectious diseases.

Keywords: Bioaerosol, Cyclone sampler, High-flow sampling, Portable sampler

\section{INTRODUCTION}

There is a long standing challenge to address the threat from the exposures to the airborne biological agents (Yao, 2018; Lioy et al., 2016; Mancon et al., 2018). This has been particularly brought to the headline in the wake of the COVID-19 pandemic (Morawska and Cao, 2020; Morawska et al., 2020; Zhang et al., 2020; Zuo et al., 2020). Airborne transmission of COVID-19 has been a subject of heated discussion and dispute lately (Marín-García et al., 2021; Galbadage et al., 2020; Gwenzi, 2020; Wong et al., 2020). Previously, as the world leading authority, the World Health Organization, has not recognized the importance of aerosol exposure of SARS-CoV-2 despite of vast amount of evidences emerging (WHO, 2020a, b; Prather et al., 2020). The dispute or discrepancy is partially due to the use of different terminologies on one hand by different disciplines (WHO, 2020a, b; Prather et al., 2020; Wilson et al., 2020); and the other hand the available qualified techniques or methods for biological aerosol studies are fewer or not readily 
available, and many of them are very outdated (Chen and Yao, 2018; Zheng et al., 2018). All these problems contribute to the difficulty of studying the critical scientific questions in bioaerosol field (Mainelis, 2020), including airborne transmission of COVID-19.

Over the past decades, there is a growing enthusiasm of investigating various aspects of bioaersols, including the efforts of sampling instrumentation. For example, various samplers such as SASS 2300 (Ma et al., 2020), Coriolis $\mu$ (Rufino de Sousa et al., 2020) and NIOSH sampler (Chen et al., 2004; Lindsley et al., 2006) were developed. Due to atmospheric dilution, airborne pathogenic species are often in low levels, and thus to capture such a low quantity of microorganisms in a short time, a high volume sampler is needed. Additionally, there is a greater need for monitoring various indoor environments for understanding the potential hidden microbial risk, and thus portability of the sampler is often required especially for public indoor spaces. Among the target microorganisms, efficient samplers for viruses are significantly lacking. Some studies for sampling airborne viruses indicated that existing samplers exhibited poor performances due to small sampling flowrates when collecting the viral particles (Prost et al., 2019; Rahmani et al., 2020). Among others, the major technological challenge when sampling airborne viruses is the size of the virus which requires a very small sampler's cutoff size in order to collect them. A smaller cutoff size is a tradeoff for a high power consumption and also the damages of the agents to be collected because the sampling is based on the particle inertia (Bekking et al., 2019). Due to this significant technological barrier, viral aerosol related work is difficult to be carried out, which partially explains the mentioned dispute for the airborne transmission of COVID-19. There is an increasing use of cyclone sampler for sampling bioaerososls (Ma et al., 2020; Lane et al., 2020; Chia et al., 2020; Wang et al., 2020). A cyclone sampler utilizes a centrifugal force to impact the particles into liquid. The cyclone sampler has already been successfully demonstrated in sampling airborne bacteria, fungi, and viruses (Cho et al., 2019; Chang et al., 2019; Guo et al., 2020). While reviewing existing cyclone samplers, most samplers have a lower sampling low rate requiring a longer sampling time, which has significant disadvantage in urgent biosafety scenarios. Importantly, for the brief sampling time such a cyclone is not capable of collecting those less frequently occurring species such as SARS-CoV-2 in the air. Besides, its influencing factors such as sampling duration and collection liquid volume were not well characterized.

Here, this work, as an effort to counter increasing airborne biological threat, was carried out to develop a portable cyclone sampler that is able to rapidly capture and concentrate low level airborne bio-agents while particularly achieving the following objectives: 1) Develop a new portable high flow cyclone sampler especially for collecting those low level of pathogens such as pathogenic viruses; 2) Characterize the performances of the developed sampler using both aerosolized and ambient particles, and further compared it with the standard liquid sampler; 3) Couple the developed sampler with a robot to achieve unmanned air sampling based on a predetermined route. The results from this work would lend significant efforts to studying aerosol transmission of infectious diseases.

\section{MATERIALS AND METHODS}

\subsection{Sampler Design}

The cyclone aerosol sampler, named as Yao-CSpler, was designed following the configurations shown in Fig. 1. It is a cyclone sampler which collects airborne particles into liquid at a sampling flow rate of $400 \mathrm{~L} \mathrm{~min}^{-1}$. The sampler was designed with optimal dimensions via simulation to achieve high performance by considering particle wall loss, collection efficiency and evaporation rate. The sampler was operated by a fan that is powered by a battery. The dimensions of the YaoCSpler are shown in Fig. 1(A), and the weight of the whole sampler is less than $4 \mathrm{~kg}$. The sampler was also designed to have a mesh filter (pore size $100 \mu \mathrm{m}$ ) installed to remove those larger particles $(>100 \mu \mathrm{m})$. When operating, the Yao-CSpler is provided with a collection liquid of 2$3 \mathrm{~mL}$ as shown in Fig. 1(B). An example of the Yao-CSpler is shown in Fig. 1(C). During the sampler operation, the fan creates a vacuum, the airborne particles are then sucked into the cyclone as shown in Fig. 1(B) as a motion of swirling toward the end of the collection vessel.

According to the research of the cyclone sampler in previous studies (Lapple, 1950; Leith and Mehta, 1967), the cutoff size $d_{50}$ can be estimated with the following equation: 
(A)

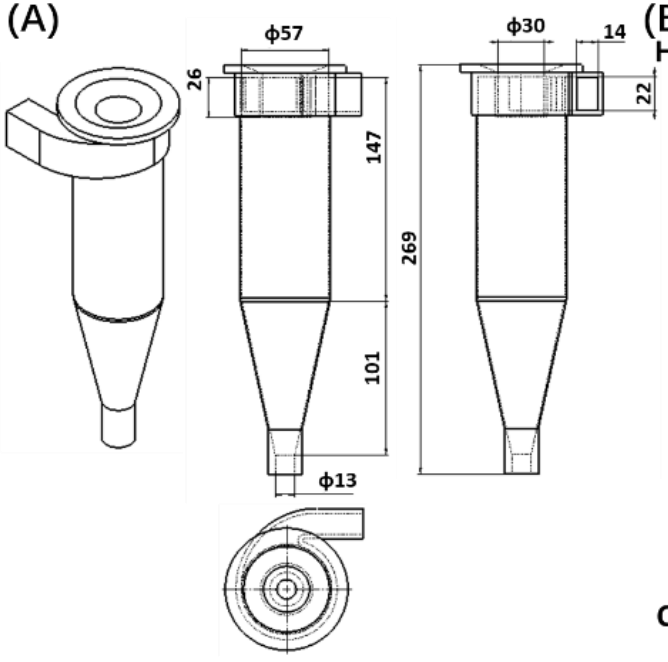

(B)

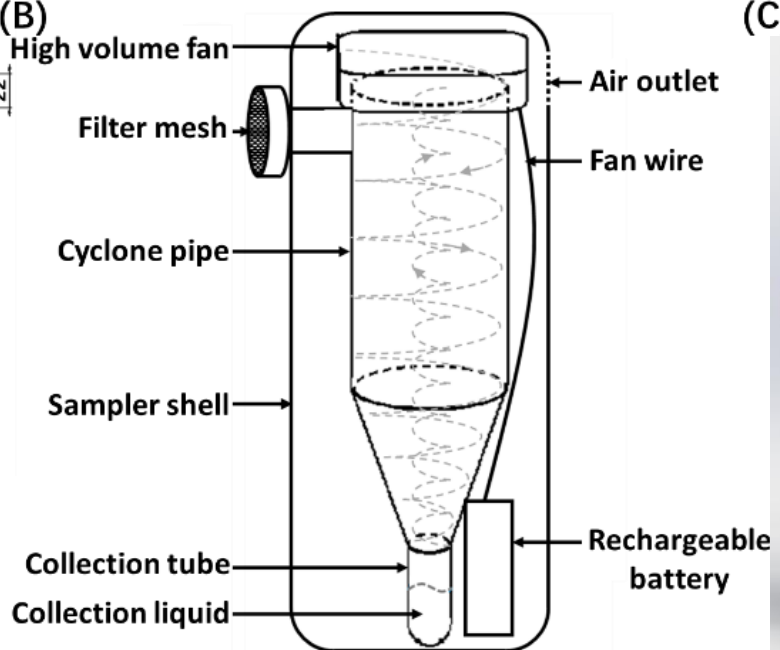

(C)

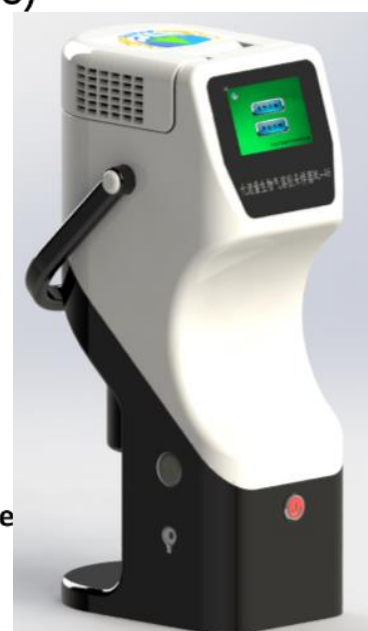

Fig. 1. Schematic diagram of the Yao-CSpler: (A) Three-view drawing and relevant dimensions of the cyclone pipe; (B) Detailed diagram of the sampler's components; (C) An example of new three-dimensional model appearance of the Yao-CSpler.

$d_{50}=3\left[\frac{\mu_{g} b}{2 \pi \rho_{p} v_{g} N_{e}}\right]^{\frac{1}{2}}$

$\mu_{g}$ is the gas viscosity, here is selected as $1.83 \times 10^{-5} \mathrm{~Pa} \cdot \mathrm{s} ; b$ is the width of gas inlet, take $14 \mathrm{~mm}$ as the dimensions marked in Fig. 1(A); $\rho_{p}$ is the particle density and take $1000 \mathrm{~kg} \mathrm{~m}^{-3}$ usually; $v_{g}$ is the gas velocity through cyclone inlet, calculated with sampling flow rate $\left(400 \mathrm{~L} \mathrm{~min}^{-1}\right)$ and gas inlet cross-sectional area $\left(14 \times 22 \mathrm{~mm}^{2}\right) ; N_{e}$ is the effective number of turns the gas makes in the cyclone, a constant value, typical of 5 .

Using the Eq. (1), the theoretical cutoff size $d_{50}$ of the Yao-CSpler is calculated as $1.84 \mu \mathrm{m}$.

\subsection{Performance Measurements of the Yao-CSpler}

\subsubsection{Experimental setup and test particles}

The experimental setup used for testing the physical performances of the Yao-CSpler is shown in Fig. 2(A). In order to determine the actual performance of the Yao-CSpler, nebulized uniform polystyrene latex (PSL) microsphere (Bangs Laboratories, Inc., Fishers, IN), Gram positive bacteria$B$. subtilis, Gram negative bacteria-P. fluorescens and indoor airborne particles were used to evaluate the physical collection efficiencies and experimental cut-off sizes of Yao-CSpler sampler. The PSL particles have mean sizes of 0.5, 0.8, 2.07, 3.11, and $5.15 \mu \mathrm{m}$ as listed in Fig. 2(B). $P$. fluorescence is often used as a sensitive bacteria to environmental stresses, while $B$. subtilis is often used as a hardy species (Liang et al., 2012). The test particles also include indoor airborne particles of $0.3-10 \mu \mathrm{m}$ (the detectable aerodynamic size range of an Optical Particle Counter) (OPC) (model 1.108; Grimm Technologies Inc., Douglasville, GA) (Fig. 2 (B)).

\subsubsection{Characterization of collection efficiencies}

As shown in Fig. 2(A), the test particles (PSL, B. subtilis and P. fluorescens) were aerosolized (at the flow rate of $2.5 \mathrm{~L} \mathrm{~min}^{-1}$ ) into the laminarizer tube by the Collison Nebulizer (BGI Inc., Waltham, $\mathrm{MA}$ ) and diluted with particles-free air to make up the difference of flow rate; meanwhile, the Yao-CSpler was placed near the outlet of the chamber to sample the airborne particles coming out of the tube. The aerosolized particle concentrations close to the inlet and outlet of the YaoCSpler were monitored by an OPC. The collection efficiencies of actual indoor airborne particles were also evaluated with the OPC. The physical collection efficiencies of the Yao-CSpler for various particles were determined using the following equation: $E=\left(1-C_{\text {outet }} / C_{\text {inlet }}\right) \times 100 \%$. The experimental cutoff size of the Yao-CSpler was also estimated using the collection efficiency curve. The 0.1$10 \mu \mathrm{m}$ particle movement trajectories inside the cyclone were simulated using ANASYS software 
(A)

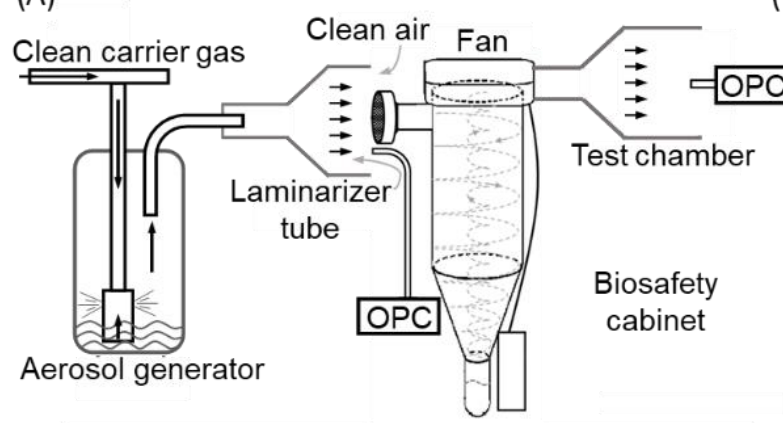

(C)

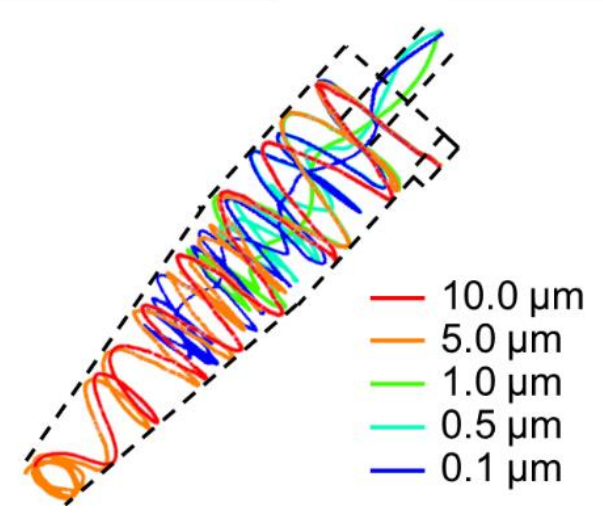

(B)

\begin{tabular}{cc}
\hline Test particles & Aerodynamic size, $\boldsymbol{\mu m}$ \\
\hline PSL & $0.5,0.8,2.07,3.11,5.15$ \\
P. fluorescens & $\sim 0.61$ \\
B. subtilis & $\sim 0.86$ \\
$\begin{array}{c}\text { Indoor airborne } \\
\text { particles }\end{array}$ & (detectable size range with OPC) \\
\hline
\end{tabular}

(D)

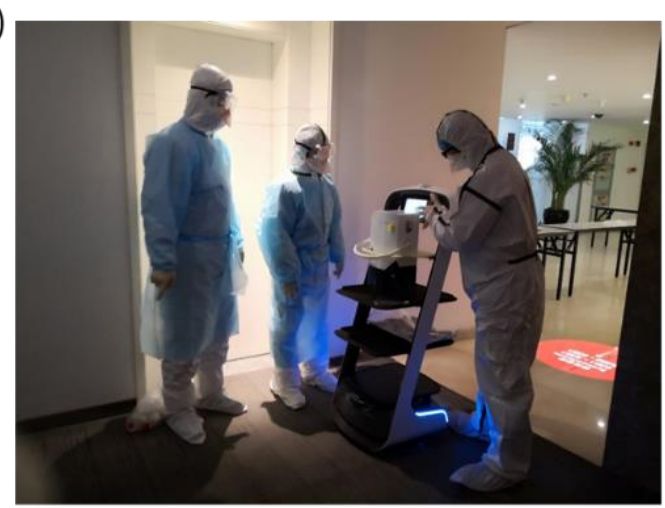

Fig. 2. Lab testing and practical applications of the Yao-CSpler: (A) Schematic diagram of the experimental setup for investigating physical collection efficiencies of the Yao-CSpler; (B) Type and size details of particles used for evaluation of the Yao-CSpler: polystyrene latex (PSL) microspheres, B. subtilis var. niger (Gram positive bacteria), P. fluorescens (Gram negative bacteria) and indoor airborne particles; (C) Simulation graph of movement trajectories of 0.1-10 $\mu \mathrm{m}$ particles inside the cyclone using ANASYS software; (D) Example of the unmanned Yao-CSpler integrated with a robot was taking air samples automatically from a quarantine hotel in Beijing.

(Fig. 2(C)). Larger particles (>5 $>\mathrm{m}$ ) were seen close to the cyclone wall and trapped on the bottom, while a few smaller particles $(\leq 1 \mu \mathrm{m})$ escaped the sampler. The detailed size-dependent particle efficiency curve is shown in Fig. 3(A).

\subsection{Bioaerosol Sampling and Evaluation Including SARS-CoV-2}

In addition to physical collection efficiencies of various particles, the collection performances of the Yao-CSpler for bioaerosols were also evaluated with both indoor and outdoor airborne particles. The concentration of culturable microorganisms obtained by plate culturing (Luria-Bertani agar plate for bacteria; Sabouraud dextrose agar for fungi) and the total bacterial concentration obtained by qPCR analysis were used to explore the effect of sampling time on the bioaerosol collection performance of the Yao-CSpler. Additionally, the survival of microorganisms under different sampling durations was studied using a BackLight kit (L-7012; Molecular Probes, Inc., Eugene, OR) staining to visually characterize the biological activity of samples collected by the Yao-CSpler. Furthermore, airborne microbial community structures in the samples from the Yao-CSpler were compared with those collected by a well-proven liquid impinging sampler-BioSampler (SKC Inc., Eighty Four, PA) under three different outdoor pollution conditions (clean, haze and dusty) given the same sampling duration. During the emergency period of COVID-19 outbreaks, the Yao-CSpler integrated with a robot was further tested for sampling airborne SARS-CoV-2 in both Wuhan and Beijing as shown in Fig. 2(D) (Zhou et al., 2020; Ma et al., 2020). The robot is a commercially available intelligent delivery robot, and the model is PuduBot (Pudu tech. Inc.). It is $516 \times 500 \times$ $1288 \mathrm{~mm}$ in machine dimensions, $35 \mathrm{~kg}$ in weight, the speed is $0.5-1.2 \mathrm{~m} \mathrm{~s}^{-1}$ and can support a maximum load of $30 \mathrm{~kg}$. It has 3D obstacle avoidance and visual positioning functions, and can travel according to the established map and bypass obstacles by itself. After simple installation and fixation, the robot assisted Yao-CSpler moved along a pre-determined route inside hospital 
or quarantine hotel as described in recent studies. The Yao-CSpler uses a high-flow fan to sample the air, and moves smoothly on a pre-set sampling route.

\subsection{Statistical Analysis}

The performances of the Yao-CSpler under different sampling times were analyzed using ANOVA or t-test following a normality test, and the diversities of bacterial structures in the air samples collected were evaluated using operational taxonomic units (OTU) (performed by usearch software, V10), Shannon index (calculated with QIIME, V1.9.1). A p-value of less than 0.05 indicates a statistically significant difference.

\section{RESULTS AND DISCUSSION}

This work designed and evaluated a new liquid cyclone sampler named as Yao-CSpler for collecting airborne bacteria, fungi and as an application for collecting airborne SARS-CoV-2 in practical environments, and its influencing factors were studied.

Fig. 3(A) shows the physical collection efficiencies of the sampler when sampling aerosolized particles and actual indoor particles. When collecting indoor airborne particles, the sampler demonstrated an experimental cutoff size of $0.58 \mu \mathrm{m}$, and for particles larger than $1 \mu \mathrm{m}$ the physical collection efficiency reached about $100 \%$. The efficiency of a cyclone sampler is related to the designed size, speed of rotation in the cyclone, temperature, humidity and air characteristics. The calculation of theoretical cutoff size $d_{50}$ in this study is based on a classical calculation method, i.e., time-of-flight theory (Lapple, 1950). Here, the inlet velocity $\left(21.7 \mathrm{~m} \mathrm{~s}^{-1}\right.$, Table 1$)$ of the Yao-CSpler is higher than $14.24 \mathrm{~m} \mathrm{~s}^{-1}$ for a typical cyclone design as described in Lapple's work (Lapple, 1950). Besides, as a non-numerical approach the time-of-flight theory has been demonstrated to show limitations in predicting of the cyclone cutoff size (Zhao and Su, 2018). A previous study also found that increasing the flowrate or velocity could improve the collection efficiency under constant cyclone design size (Sigaev et al., 2006). The variations in cyclone design could contribute to the differences between theoretical prediction and experimental measurement. As observed from the figure, the collection efficiencies varied slightly for different types of particles, although they had similar sizes, which might be due to different surface characteristics of the particles that could impact the interaction of the particles and the collection media. For example, the morphology, hydrophobic or hydrophilic features will influence the physical collection efficiencies, cutoff size, viability of collected bio-agents (Su et al., 2019; Matthew et al., 2008; Viswanathan et al., 2017). For the aerosolized particles, most of them were surrounded by water droplets, thus they are more likely to be adhered to the inner wall of the sampler or aggregated with other particles into larger ones and retained by the sampler. Nonetheless, those droplets could deposit on the cyclone walls and later descended into the collection liquid due to the gravity and air stream blow. So their corresponding collection efficiencies were also higher as observed from Fig. 3(A). The collection liquid loss also affects sampling efficiency, and it depends on the distance between the downstream air outlet of the cyclone pipe and the collection liquid surface (Jet-to-liquid). The shorter the distance, the more intense the collection liquid whirl, and higher liquid loss was observed. So, too short distance may cause liquid splashing and additional loss as Fig. S1 shown. A distance of $28 \mathrm{~mm}$ seemed to be a critical value at which liquid loss remained relatively stable at a rate of $42 \mu \mathrm{L} \mathrm{min}{ }^{-1}$, and according to the regression fitting in Fig. S2, the minimum evaporation rate (without liquid splash) should be $36 \mu \mathrm{L} \mathrm{min}^{-1}$. The temperature and environment humidity would also influence the evaporation speed (Kim et al., 2007). The liquid loss was measured at $23^{\circ} \mathrm{C}$ and $60 \%$ relative humidity $(\mathrm{RH})$ in this study.

When collecting indoor airborne particles for bacteria and fungi culturing, two-minute sampling seemed to have the best performance in measuring the culturable concentration of bacteria as shown in Fig. 3(B). But the efficiency decreased when the sampling time increased to 5 minutes or longer. As for the fungal concentration, 1 minute seemed to be optimal and the efficiency also decreased when increasing the sampling time. The culturable fungal concentrations were much lower than those of bacteria for any sampling durations tested. Likewise, for outdoor environments, the similar findings were observed in Fig. 3(C): two-minute sampling was optimal for measuring the culturable bacteria concentration, and the efficiency decreased sharply with increasing 
(A)

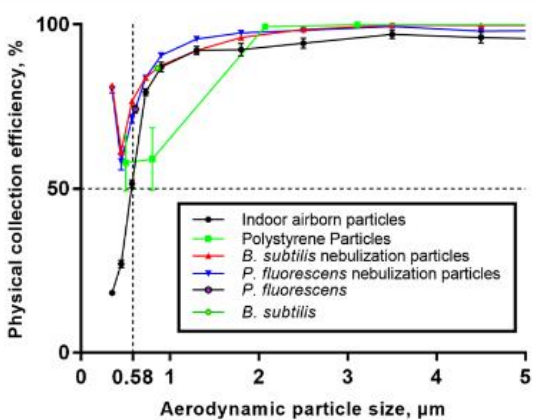

(C)

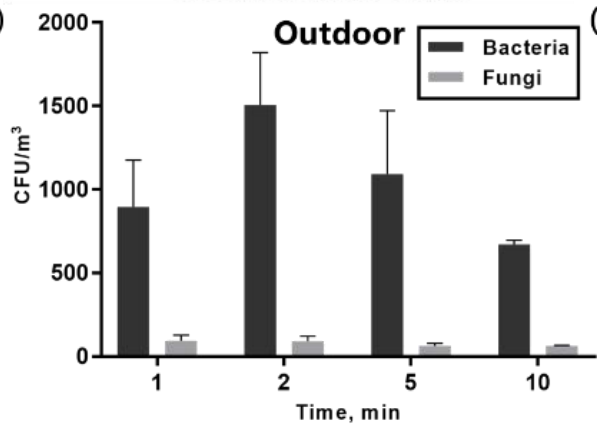

(B)

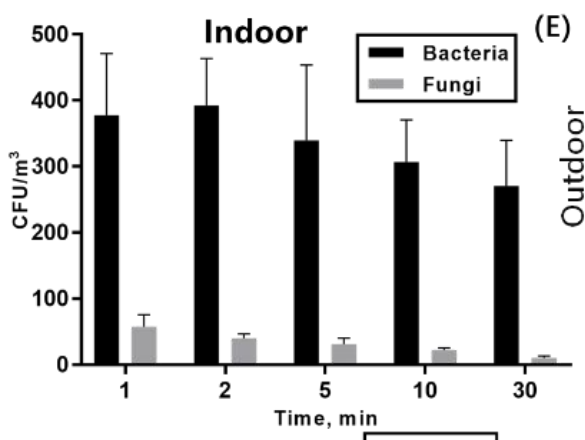

(D)

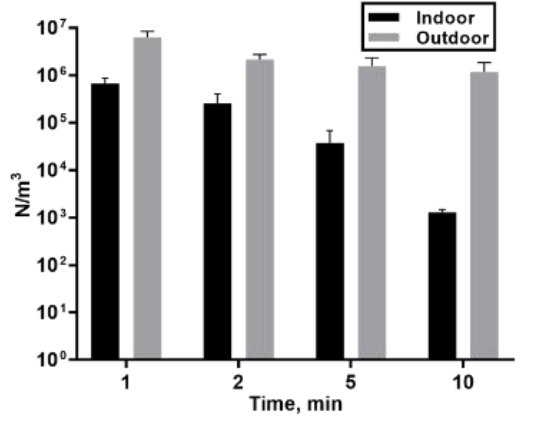

(E)
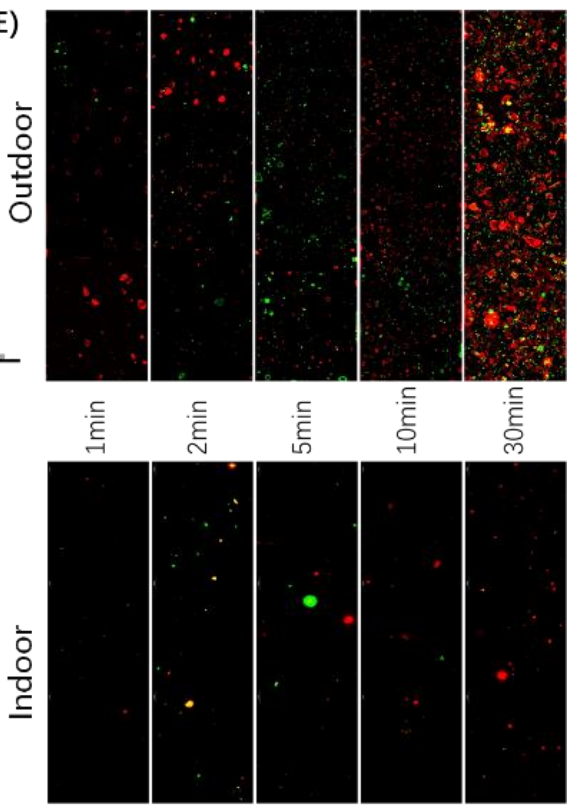

Fig. 3. (A) Physical collection efficiencies curves of the Yao-CSpler when collecting polystyrene latex (PSL) microspheres with different sized (mean particle sizes: $0.5,0.8,2.07,3.11$, and $5.15 \mu \mathrm{m}$ ), indoor airborne particles and also nebulized bacteria: $P$. fluorescence, B. subtilis; (B) and (C) Culturable microbes concentration, measured by the Yao-CSpler, for the different sampling durations both indoors and outdoors; (D) Total bacterial concentration sampled by the Yao-CSpler from both indoors and outdoors over the sampling durations (1, 2, 5, 10 minute); (E) DNA staining cells' photos of lived (green dots) or dead (red dots) bacteria particles in the Yao-CSpler's test-samples with different sampling durations (1, 2, 5, 10, 30 minutes) indoors and outdoors, respectively. All the data shown in (A)-(D) represent averages from at least three repeats and error bars stand for the standard error.

sampling time. When measuring the total bacterial load in the air using the qPCR, the results in Fig. 3(D) showed that one-minute sampling might be optimal both for both outdoor and indoor. The outdoor bacterial levels were found to be significantly higher than indoor ones, and the total concentrations also decreased with increasing sampling time. Fig. 3(E) showed that for the same sampling time outdoor bacterial levels were significantly higher than those detected indoors. And the proportion of dead bacteria (red dots) increased as the sampling time increased, which indicated that the viability of the collected airborne bacteria decreased when the sampling time increased. During the operation of the Yao-CSpler, it takes tens of seconds for the fan to achieve a preset sampling velocity $\left(400 \mathrm{~L} \mathrm{~min}^{-1}\right)$, thus the actual collected air volumes were smaller than those used for calculation. However, all the culturable microbial concentrations and total bacteria concentrations were calculated using the theoretical air volume sampled during the corresponding period, this deviation could be amplified especially for shorter sampling process. This can on the other hand explain the obtained lower levels for the first one minute sampling. As for the sharper drop of indoor total bacteria concentration, it was probably because the sampler also served as an efficient air cleaner in the non-ventilated basement laboratory where the experiments were conducted. Thus over the time the indoor particle concentration decreased substantially. Conversely, the total concentration of outdoor bacteria decreased slightly with the increase of sampling time, it indicated that the Yao-CSpler can maintain a stable total microbial collection efficiency in outdoor or open spaces. Further, with increasing sampling time, the collection liquid loss, particle bounce and reaerosolization problems occurred altogether (Riemenschneider et al., 2010). These influencing factors contributed to the lower bioaerosol levels observed in longer sampling duration as shown in Figs. 3(B-D). Especially for the concentration of culturable microorganisms, the stronger reduction of outdoor bacterial concentration in Fig. 3(C) than Fig. 3(D) suggests that a longer sampling period may damage the activity of microorganisms in the samples (Fig. 3(E) also supports this finding). In addition, regardless the efficiency advantage of short-time sampling 
as manifested from Figs. 3(B-D), the temporary background bioaerosol concentration changes caused by natural ambient air-flow could increase the uncertainty of short-term sampling (<5 minutes) (Cox et al., 2020; Grinshpun et al., 2016). Therefore, the sampling duration of the Yao-CSpler should be carefully selected to meet specific research purposes. So if the focus of the work is on the culturability of biological aerosols, a shorter sampling duration (less than 5 minutes) should be selected; otherwise the detection of less frequent species is desired, a longer sampling duration (e.g., 20-30 minutes) should be used. However, this has not been performed for viral particle recovery in this work.

The sampling flowrate $\left(400 \mathrm{~L} \mathrm{~min}^{-1}\right)$ of Yao-CSpler is about 32 times of a well-proven liquid impinging sampler named BioSampler $\left(12.5 \mathrm{~L} \mathrm{~min}^{-1}\right)$. In addition, the volume of collection liquid $(20 \mathrm{~mL})$ used by BioSampler is also 6-10 times of that using Yao-CSpler (2-3 mL). Thus, it will take much longer time for the BioSampler to obtain the same amount of air samples. For lower viral level, the collected samples by the BioSampler using the same sampling time might not be enough for PCR detection. The Yao-CSpler obtained more diversities of bacteria than the BioSampler in outdoor environments. As shown in Fig. 4(A), outdoor bacterial OTU number obtained by the YaoCSpler were 2-4 times those obtained by the BioSampler. Regardless of $\mathrm{PM}_{2.5}$ pollution conditions, the bacterial structures obtained by both samplers differed substantially as observed in Fig. 4(B). For clean and haze days, the recovered top 3 species using the BioSampler were Elizabethkingia ( $17.4 \%$ on the clean day and $17.8 \%$ on the haze day), Pseudomonas ( $8.2 \%$ on both the clean and haze days), and Acinetobacter (5.4\% on the clean day and $7.2 \%$ on the haze day); while for the dusty day the Enterobacter was the dominant species $(73.7 \%)$, followed by BurkholderiaCaballeronia-Paraburkholderia (BCP) (5.0\%) and Bacillus (2.6\%). In contrast, using the Yao-CSpler, the top 3 species on the clean day were Sphingomonas (5.8\%), Pseudarthrobacter (2.9\%), and Planococcus (2.6\%), on the haze day the top 3 species were Pseudarthrobacter (7.9\%), Planococcus (6.7\%), Kocuria (4.6\%), and for the dusty day they were Planococcus (12.4\%), Kocuria (8.2\%), and Pseudarthrobacter (7.2\%). The detailed abundances of different species were listed in Supporting File (Table S3). Given the same sampling time, the Shannon indexes for the air samples collected by the Yao-CSpler were significantly higher than those of the samples collected by the BioSampler (Fig. S3). In addition to the sampling environmental bacteria and fungi, the Yao-CSpler was also
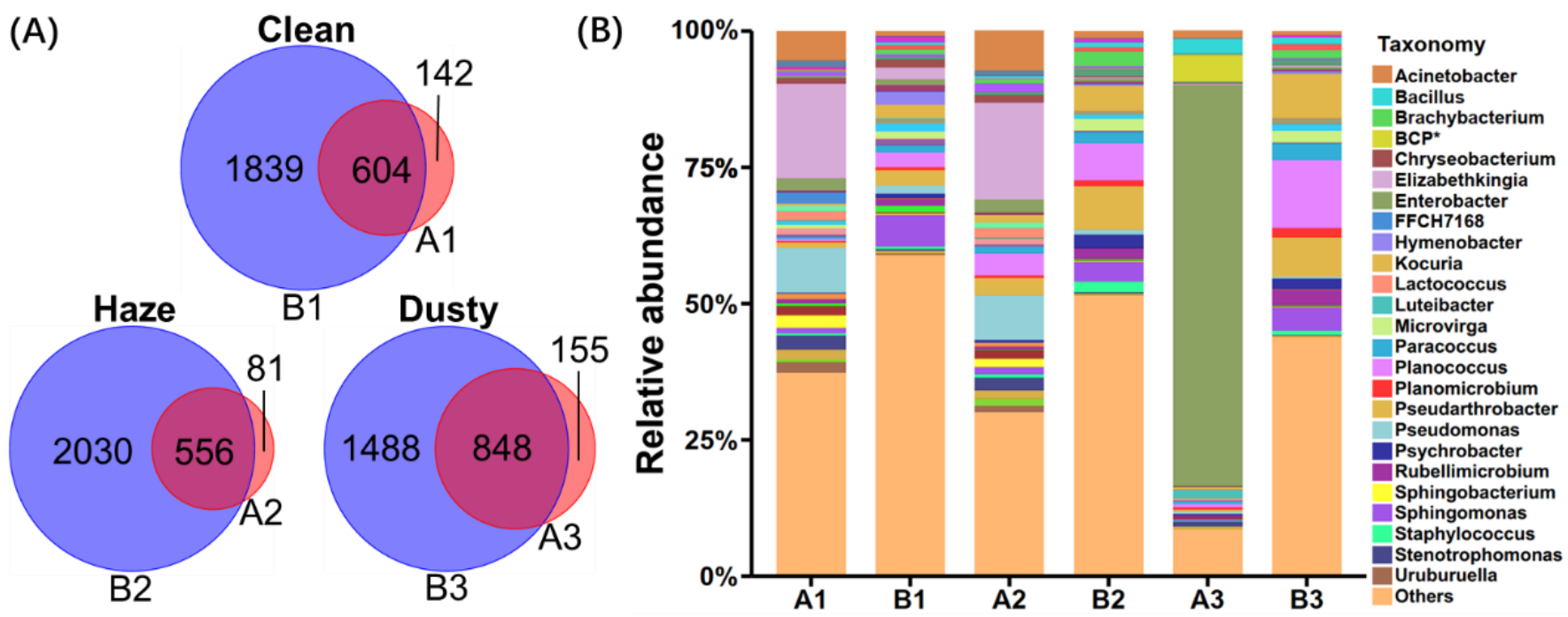

Fig. 4. Differences in community structures of bacterial aerosols between the samples collected by the BioSampler (SKC) and the Yao-CSpler respectively under different polluted outdoor conditions. (A) The Venn diagram of OTUs structures between the samples collected by the Yao-CSpler and the BioSampler under the same sampling time. (B) The relative abundances of the top 25 species in the samples collected by the BioSampler and the Yao-CSpler (at the genus level). The labels A1, A2 and A3 represent the samples collected by the BioSampler on clean, haze and dusty days, respectively. B1, B2 and B3 represent the samples collected on the same corresponding clean, haze and dusty days using the Yao-CSpler, respectively. The meteorological and pollutant concentration information during the sampling periods were listed in Tables S1 and S2. BCP ${ }^{*}$ represents BurkholderiaCaballeronia-Paraburkholderia. 
used in monitoring SARS-CoV-2 in recent two studies (Zhou et al., 2020; Ma et al., 2020). Particularly, it was shown in Wuhan hospitals (Fig. 2(D)) that the air samples collected using the Yao-CSpler contained 9-219 SARS-CoV-2 RNA copies per $\mathrm{m}^{3}$ (Zhou et al., 2020). In their study, digital PCR (dPCR) was used to detect the absolute quantitative airborne SARS-Cov-2, and experimental details can be found from the work. After sampling, 1-2 $\mathrm{mL}$ collection liquid remained and was used for extraction of RNA directly without further sample enrichment. The concentration of SARS-Cov-2 in air $\left(C_{\text {air }}\right)$ was averaged over the entire sampling time period and calculated approximately with the following Eq. (2):

$$
C_{\text {air }}=\frac{C_{R N A} v_{\text {sample }}}{Q t_{\text {sample }}}
$$

$C_{R N A}$ is the absolute quantitative SARS-Cov-2 RNA concentration in the sample (copies $\mathrm{mL}^{-1}$ ); $v_{\text {sample }}$ is the volume of sample collection liquid $(\mathrm{mL}) ; Q$ is the flowrate of the YAO-CSpler (400 $\left.\mathrm{L} \mathrm{min}^{-1}\right) ; t_{\text {sample }}$ is the sampling duration ( $\mathrm{min}$ ).

As for using the Eq. (2), there might be an underestimate of the actual airborne viral level. Firstly, the physical collection efficiency for viral size particle could be low, thus failing to collect all of them. Secondly, during the sampling process the collected viral particles could be damaged due to desiccation and mechanical stress arising from high wind speed and collision speed, thus leading to RNA loss. Lastly, the collected particles could be re-aerosolized. For 40 minutes' sampling, the collection liquid volume was observed to have decreased from $3 \mathrm{~mL}$ to $1-2 \mathrm{~mL}$ depending on the actual environmental humidity levels. It is difficult to set a detectable level of airborne COVID-19 virus since the sampling time could be different. If excessive evaporation is minimized, sampling for 40 min or shorter is preferred. Accordingly, use of Eq. (2) would yield an underestimate of actual airborne viral level.

These data show that the Yao-CSpler can collect not only airborne bacteria and fungi, but also viruses such as SARS-CoV-2 that had lower levels in open space air. The cutoff size of the sampler is $0.58 \mu \mathrm{m}$, which means the sampler has at least $50 \%$ of collection efficiency for particles of larger than $0.58 \mu \mathrm{m}$. For single viral particle, the sampler might have very low collection efficiency. However, in practical environments, those nano viruses are easily attached to larger particles or agglomerate with each other to form larger particles. Thus, the developed sampler is suitable for rapid virus detection. Nonetheless, some single viral particles could escape the system, not being collected, thus leading to a possible underestimate of the viral load. Decreasing the cutoff size would require a substantial increase of sampling flow rate, which could represent a significant challenge to the portability and liquid evaporation. The BioSampler has a lower cutoff size of $0.3 \mu \mathrm{m}$, however its sampling flow rate is $12.5 \mathrm{~L} \mathrm{~min}^{-1}$. For low level pathogen in the air, e.g., COVID-19 virus, much longer time is needed to obtain detectable viral samples. The sampling velocity of the BioSampler is much higher, $\sim 300 \mathrm{~m} \mathrm{~s}^{-1}$ by calculation, which could damage the properties of biological cells. In contrast, the contour velocity of the Yao-CSpler ranges from $26.8-44.6 \mathrm{~m} \mathrm{~s}^{-1}$, and axial velocity ranges from $6.42-32.3 \mathrm{~m} \mathrm{~s}^{-1}$. Thus, the velocity component of the developed sampler is much smaller than that of the BioSampler.

Over the past decades, a significant amount of time was devoted to the field of bioaerosol sampling, but compared with other environmental disciplines relevant number of scientists in this field is strikingly fewer. Sampling is the first step in studying bioaerosol, and plays a critical role in the outcome of the investigation. In face of the COVID-19 pandemic, it seems that a great number of efforts are moving toward this research direction (Seo et al., 2020; Ong et al., 200). But among other agents, sampling viruses is typically challenging. In addition to its small size, preserving its viability is difficult for further infectivity confirmation (Seo et al., 2020; Oppliger et al., 2011; Lindsley et al., 2017; Fabian et al., 2009). In a previous study, a filtering method-MD8 Airscan (Sartorius Stedim Biotech GmbH, Goettingen, Germany) together with gelatin filter ( $3 \mu \mathrm{m}$ pore size) has been used for sampling of MERS-coronavirus (MERS-CoV) and SARS-CoV viruses in indoor air of hospitals (Zhao et al., 2014; Kim et al., 2016). The gelatin filter can easily dissolve into water, and recently it was used together with a cassette for sampling SARS-CoV-2 (Liu et al., 2020). Fabian et al. (2009) compared four different samplers for collecting airborne influenza virus, and they found that only the SKC Biosampler was capable of efficiently collecting airborne virus and 
Table 1. Physical characteristics of the Yao-CSpler and other cyclone samplers.

\begin{tabular}{|c|c|c|c|c|c|c|}
\hline $\begin{array}{l}\text { Cyclone } \\
\text { Name }\end{array}$ & $\begin{array}{l}\text { Flowrate } \\
\left(\mathrm{L} \mathrm{min}^{-1}\right)\end{array}$ & $\begin{array}{l}\text { Inlet Velocity } \\
\left(\mathrm{m} \mathrm{s}^{-1}\right)\end{array}$ & $\begin{array}{l}\text { Theoretical } d_{50} \\
(\mu \mathrm{m})\end{array}$ & $\begin{array}{l}\text { Experimental } d_{50} \\
(\mu \mathrm{m})\end{array}$ & $\begin{array}{l}\text { Reynolds } \\
\text { number }\end{array}$ & References \\
\hline Yao-CSpler & 400 & 21.7 & 1.84 & 0.58 & 24682 & This work \\
\hline $\mathrm{NIOSH}$ & 3.5 & $18.6^{\#}$ (stage 1$)$ & $0.74^{\#}$ (stage 1$)$ & 1.8 (stage 1$)$ & $2480^{\#}$ (stage 1 ) & Lindsley et al., 2006 \\
\hline BC-251 & & $44.0^{\#}$ (stage 2 ) & $0.39^{\#}$ (stage 2 ) & 1 (stage 2) & $3813^{\#}$ (stage 2) & \\
\hline SASS 2300 & 325 & / & / & $>1$ & / & Dybwad et al., 2014 \\
\hline $\mathrm{NIOSH}$ & $2 / 4$ & $10.8\left(2 \mathrm{~L} \mathrm{~min}^{-1}\right)$ & 1.3 & $2.5\left(2 \mathrm{~L} \mathrm{~min}^{-1}\right)$ & $1450\left(2 \mathrm{~L} \mathrm{~min}^{-1}\right)$ & Chen et al., 2004 \\
\hline BC-112 & & $21.4\left(4 \mathrm{~L} \mathrm{~min}^{-1}\right)$ & $\left(2 \mathrm{~L} \min ^{-1}\right)$ & $1.5\left(4 \mathrm{~L} \mathrm{~min}^{-1}\right)$ & $2863\left(4 \mathrm{~L} \mathrm{~min}^{-1}\right)$ & \\
\hline Coriolis $\mu$ & 300 & / & / & $>1$ & / & Carvalho et al., 2008 \\
\hline WWC-1250 & 1250 & 52.8 & / & 1 & 21200 & McFarland et al., 2010 \\
\hline
\end{tabular}

\#: Calculated according to the parameters provided in the literatures. /: No information provided from literatures.

preserving their infectivity. However, they also pointed out that when collecting naturally occurring aerosols the SKC Biosampler exhibited declining collection efficiency for smaller particles, high dilution factor, and low sampling flow rate problems. A lot of cyclone samplers have also been developed for collecting bioaerosol particles as shown in Table 1, and most of them have not been characterized thoroughly (Mbareche et al., 2018). Compared to filtration-based and impactionbased sampler, the liquid impinger represented by cyclone samplers can reduce the desiccation, particle bounce, and impaction stress, thus substantially improving the biological collection efficiencies (Verreault et al., 2008). Besides, liquid samples can be immediately used with nucleic acid and immunological methods for fast biological analysis. This eliminates the need of the extraction of biological cells from a filter if otherwise used. For culture-based impaction method, it takes several days to cultivate the collected particles, and for some non-culturable ones the method cannot detect them. Liquid-impingement based methods offer promising solutions for sampling airborne biological agents, however its problems remain ahead. For example, high volume sampling would lead to fast loss of collection liquid (thus a decline in efficiency), impaction of biological cells, and reaerosolization of already collected particles over longer sampling. For larger particles, there are also significant loss on the cyclone wall. In the future, mineral oil, a nonevaporating material, can be tested as a collection medium. For persevering viral agents, a cooling module can be also equipped with high volume sampling. Verreault et al. (2008) extensively reviewed methods of airborne virus sampling such as liquid impingers, solid impactors, filters, and electrostatic precipitators by analyzing more than 100 papers, and they concluded that the a variety of environmental and methodological factors not only influence the their efficiencies, but also affect the structure integrity of the virus collected. In addition to considering these parameters, future bioaerosol sampler would be more intelligent, i.e., being coupled with various environmental sensors connected to the samples collected, self-moving, and remotely controllable. With such active efforts, a better understanding of airborne transmission of infectious diseases can be only obtained.

\section{CONCLUSIONS}

Here, we have designed and evaluated a new cyclone sampler named Yao-CSpler which has a sampling flowrate of $400 \mathrm{~L} \mathrm{~min}^{-1}$. It achieves an experimental particle cutoff size of $0.58 \mu \mathrm{m}$. Experimental data show that the sampler could efficiently collect airborne bacteria, fungi, and also viruses such as SARS-CoV-2. In addition, we have also found that the Yao-CSpler achieved an optimal performance at a Jet-to-liquid of $28 \mathrm{~mm}$ (from air stream exit to the surface of the collection liquid) at room temperature $\left(23^{\circ} \mathrm{C}\right)$ and $60 \% \mathrm{RH}$ yet with a lower and stable liquid loss rate. Besides, we also found that the Yao-CSpler outperformed the BioSampler when sampling outdoor bacterial aerosols for the same amount of time under different $\mathrm{PM}_{2.5}$ pollution conditions in terms of bacterial diversity and species richness. This might be largely due to its high sampling flow rate which recovered less frequently occurring species from the air and its sampling enrichment capacity to allow the air samples to be detectable (Zhao et al., 2014). The BioSampler has a sampling flow rate of $12.5 \mathrm{~L} \mathrm{~min}^{-1}$, and it collects $750 \mathrm{~L}$ air per hour; while the Yao-CSpler 
collects 30 times more air per unit of time. The BioSampler is impacted more by environmental fluctuations, e.g., concentration level, wind direction change; while the Yao-CSpler is relatively less impacted by such changes. For quantification, it is desired that the air sampling time should be longer to be more representative, e.g., when coupled with nucleic acid method; however long sampling time would cause viability loss if otherwise culturing method is applied. Therefore, optimal sampling time should be chosen according to specific requirements of an airborne agent investigation. In terms with sampling airborne virus, the collection efficiency could be low compared to larger particles. The sampler integrated with a robot was demonstrated to be capable of moving stably along the pre-set route in a typical environment for continuous air sampling.

\section{ACKNOWLEDGMENTS}

This research was supported by the NSFC Distinguished Young Scholars Fund Awarded to M. Yao (21725701) and a National Natural Science Foundation of China (NSFC) grants (22040101). This work was also partially support by a grant from Guangzhou Laboratory (EKPG21-02). The authors also thank a company in Beijing for their assistance with the sampler physical assembly.

\section{SUPPORTING INFORMATION}

Supporting Information: Meteorological information of three different pollution dates, Ambient Air Quality Information (AQI) and main pollutant concentration of three different pollution dates, the details of relative abundances of bacterial species recovered by different samplers; Liquid evaporation as well as the rate per min, and Shannon index for the bacterial communities recovered.

\section{SUPPLEMENTARY MATERIAL}

Supplementary material for this article can be found in the online version at https://doi. org/10.4209/aaqr.210130

\section{REFERENCES}

Bekking, C., Yip, L., Groulx, N., Doggett, N., Finn, M., Mubareka, S. (2019). Evaluation of bioaerosol samplers for the detection and quantification of influenza virus from artificial aerosols and influenza virus-infected ferrets. Influenza Other Respir. Viruses 13, 564-573. https://doi.org/1 $0.1111 /$ irv.12678

Carvalho, E., Sindt, C., Verdier, A., Galan, C., O'Donoghue, L., Parks, S. (2008). Performance of the Coriolis air sampler, a high-volume aerosol-collection system for quantification of airborne spores and pollen grains. Aerobiologia 24, 191-201. https://doi.org/10.1007/s10453-008-9098-y

Chang, C.W., Ting, Y.T., Horng, Y.J. (2019). Collection efficiency of liquid-based samplers for fungi in indoor air. Indoor Air 29, 380-389. https://doi.org/10.1111/ina.12535

Chen, B.T., Feather, G.A., Maynard, A., Rao, C.Y. (2004). Development of a personal sampler for collecting fungal spores. Aerosol Sci. Technol. 38, 926-937. https://doi.org/10.1080/02786829 0511218

Chen, H., Yao, M. (2018). A high-flow portable biological aerosol trap (HighBioTrap) for rapid microbial detection. J. Aerosol Sci. 117, 212-223. https://doi.org/10.1016/j.jaerosci.2017.11.012

Chia, P.Y., Coleman, K.K., Tan, Y.K., Ong, S.W.X., Gum, M., Lau, S.K. (2020). Detection of air and surface contamination by SARS-CoV-2 in hospital rooms of infected patients. Nat. Commun. 11, 1-7. https://doi.org/10.1038/s41467-020-16670-2

Cho, Y.S., Hong, S.C., Choi, J., Jung, J.H. (2019). Development of an automated wet-cyclone system for rapid, continuous and enriched bioaerosol sampling and its application to real-time detection. Sens. Actuators, B 284, 525-533. https://doi.org/10.1016/j.snb.2018.12.155

Cox, J., Mbareche, H., Lindsley, W.G., Duchaine, C. (2020). Field sampling of indoor bioaerosols. Aerosol Sci. Technol. 54, 572-584. https://doi.org/10.1080/02786826.2019.1688759 
Dybwad, M., Skogan, G., Blatny, J.M. (2014). Comparative testing and evaluation of nine different air samplers: End-to-end sampling efficiencies as specific performance measurements for bioaerosol applications. Aerosol Sci. Technol. 48, 282-295. https://doi.org/10.1080/02786826. 2013.871501

Fabian, P., McDevitt, J.J., Houseman, E.A., Milton, D.K. (2009). Airborne influenza virus detection with four aerosol samplers using molecular and infectivity assays: Considerations for a new infectious virus aerosol sampler. Indoor Air 19, 433. https://doi.org/10.1111/j.1600-0668.2009. 00609.x

Galbadage, T., Peterson, B.M., Gunasekera, R.S. (2020). Does COVID-19 spread through droplets alone? Front. Public Health 8, 163. https://doi.org/10.3389/fpubh.2020.00163

Grinshpun, S.A., Buttner, M.P., Mainelis, G., Willeke, K. (2016). Sampling for airborne microorganisms, in: Manual of Environmental Microbiology, John Wiley \& Sons, Ltd, pp. 3.2.21-3.2.2-17. https://doi.org/10.1128/9781555818821.ch3.2.2

Guo, Z. D., Wang, Z. Y., Zhang, S. F., Li, X., Li, L., Li, C. (2020). Aerosol and surface distribution of severe acute respiratory syndrome coronavirus 2 in hospital wards, Wuhan, China, 2020. Emerging Infect. Dis. 26, 1583-1591. https://doi.org/10.3201/eid2607.200885

Gwenzi, W. (2020). Leaving no stone unturned in light of the COVID-19 faecal-oral hypothesis? A water, sanitation and hygiene (WASH) perspective targeting low-income countries. Sci. Total Environ. 753, 141751. https://doi.org/10.1016/j.scitotenv.2020.141751

Hsiao, T.C., Huang, S.H., Hsu, C.W., Chen, C.C., Chang, P.K. Effects of the geometric configuration on cyclone performance. J. Aerosol Sci. 2015, 86, 1-12. https://doi.org/10.1016/j.jaerosci.201 5.03 .005

Kim, S.H., Chang, S.Y., Sung, M., Park, J.H., Bin Kim, H., Lee, H. (2016). Extensive viable Middle East respiratory syndrome (MERS) coronavirus contamination in air and surrounding environment in MERS isolation wards. Rev. Infect. Dis. 63, 363-369. https://doi.org/10.1093/cid/ciw239

Kim, S.W., Ramakrishnan, M.A., Raynor, P.C., Goyal, S.M. (2007). Effects of humidity and other factors on the generation and sampling of a coronavirus aerosol. Aerobiologia 23, 239-248. https://doi.org/10.1007/s10453-007-9068-9

Lane, M.A., Brownsword, E.A., Morgan, J.S., Babiker, A., Vanairsdale, S.A., Lyon, G.M. (2020). Bioaerosol sampling of a ventilated patient with COVID-19. Am. J. Infect. Control 48, 15401542. https://doi.org/10.1016/j.ajic.2020.07.033

Lapple, C.E. (1950). Gravity and centrifugal separation. Am. Ind. Hyg. Assoc. Q. 11, 40-48. https://doi.org/10.1080/00968205009344283

Leith, D., Mehta, D. (1973). Cyclone performance and design. Atmos. Environ. 7, 527-549. https://doi.org/10.1016/0004-6981(73)90006-1

Liang, Y., Wu, Y., Sun, K., Chen, Q., Shen, F., Zhang, J. (2012). Rapid inactivation of biological species in the air using atmospheric pressure nonthermal plasma. Environ. Sci. Technol. 46, 3360-3368. https://doi.org/10.1021/es203770q

Lindsley, W.G., Green, B.J., Blachere, F.M., Martin, S.B., Law, B.F., Jensen, P.A., Schafer, M. (2017). Sampling and characterization of bioaerosols. NIOSH manual of analytical methods. 5th ed. National Institute for Occupational Safety and Health Perss, Cincinnati, $\mathrm{OH}$.

Lindsley, W.G., Schmechel, D., Chen, B.T. (2006). A two-stage cyclone using microcentrifuge tubes for personal bioaerosol sampling. J. Environ. Monit. 8, 1136-1142. https://doi.org/10.1039/B6 09083D

Lioy, P.J., Laskin, J.D., Georgopoulos, P.G. (2016). Preparedness and response to chemical and biological threats: the role of exposure science. Ann. N. Y. Acad. Sci. 1378, 108-117. https://doi.org/10.1111/nyas.13173

Liu, Yuan, Ning, Z., Chen, Y., Guo, M., Liu, Yingle, Gali, N.K., Sun, L., Duan, Y., Cai, J., Westerdahl, D., Liu, X., Xu, K., Ho, K., Kan, H., Fu, Q., Lan, K. (2020). Aerodynamic analysis of SARS-CoV-2 in two Wuhan hospitals. Nature 582, 557-560. https://doi.org/10.1038/s41586-020-2271-3

Ma, J., Qi, X., Chen, H., Li, X., Zhang, Z., Wang, H. (2020). Coronavirus disease 2019 patients in earlier stages exhaled millions of severe acute respiratory syndrome coronavirus 2 per hour. Clin. Infect. Dis. ciaa1283 https://doi.org/10.1093/cid/ciaa1283

Ma, X., Fang, Z., Li, F., Hu, K. (2020). Determination of performance-parameter design and impact factors of sampling efficiency for bioaerosol cyclones. Biotechnol. Biotechnol. Equip. 34, 640651. https://doi.org/10.1080/13102818.2020.1797529 
Mainelis, G. (2020). Bioaerosol sampling: Classical approaches, advances, and perspectives. Aerosol Sci. Technol. 54, 496-519. https://doi.org/10.1080/02786826.2019.1671950

Mancon, A., Mileto, D., Gismondo, M.R. (2018). The Global Threats from Naturally Occurring Infectious Diseases, in: Radosavljevic, V., Banjari, I., Belojevic, G. (Eds.), Defence Against Bioterrorism, Springer Netherlands, Dordrecht, pp. 13-24. https://doi.org/10.1007/978-94024-1263-5_3

Marín-García, D., Moyano-Campos, J.J., Bienvenido-Huertas, J.D. (2021). Distances of transmission risk of COVID-19 inside dwellings and evaluation of the effectiveness of reciprocal proximity warning sounds. Indoor Air 31, 335-347. https://doi.org/10.1111/ina.12738

Matthew, B.M., Middlebrook, A.M., Onasch, T.B. (2008). Collection efficiencies in an aerodyne aerosol mass spectrometer as a function of particle phase for laboratory generated aerosols. Aerosol Sci. Technol. 42, 884-898. https://doi.org/10.1080/02786820802356797

Mbareche, H., Veillette, M., Bilodeau, G.J., Duchaine, C. (2018). Bioaerosol sampler choice should consider efficiency and ability of samplers to cover microbial diversity. Appl. Environ. Microbiol. 84, e01589-18. https://doi.org/10.1128/AEM.01589-18

McFarland, A.R., Haglund, J.S., King, M.D., Hu, S., Phull, M.S., Moncla, B.W. (2010). Wetted wall cyclones for bioaerosol sampling. Aerosol Sci. Technol. 44, 241-252. https://doi.org/10.1080/0 2786820903555552

Morawska, L., Cao, J. (2020). Airborne transmission of SARS-CoV-2: The world should face the reality. Environ. Int. 139, 105730. https://doi.org/10.1016/j.envint.2020.105730

Morawska, L., Tang, J.W., Bahnfleth, W., Bluyssen, P.M., Boerstra, A., Buonanno, G. (2020). How can airborne transmission of COVID-19 indoors be minimised? Environ. Int. 142, 105832. https://doi.org/10.1016/j.envint.2020.105832

Ong, S.W.X., Tan, Y.K., Chia, P.Y., Lee, T.H., Ng, O.T., Wong, M.S.Y., Marimuthu, K. (2020). Air, surface environmental, and personal protective equipment contamination by severe acute respiratory syndrome coronavirus 2 (SARS-CoV-2) from a symptomatic patient. JAMA 323, 1610-1612. https://doi.org/10.1001/jama.2020.3227

Oppliger, A., Masclaux, F.G., Niculita-Hirzel, H. (2011). Assessment of airborne microorganisms by real-time PCR: Optimistic findings and research challenges. Front. Biosci., Scholar Ed. 3, 445453. https://doi.org/10.2741/s163

Prather, K.A., Marr, L.C., Schooley, R.T., McDiarmid, M.A., Wilson, M.E., Milton, D.K. (2020). Airborne transmission of SARS-CoV-2. Science 370, 303-304. https://doi.org/10.1126/science. abf0521

Prost, K., Kloeze, H., Mukhi, S., Bozek, K., Poljak, Z., Mubareka, S. (2019). Bioaerosol and surface sampling for the surveillance of influenza A virus in swine. Transboundary Emerging Dis. 66, 1210-1217. https://doi.org/10.1111/tbed.13139

Rahmani, A.R., Leili, M., Azarian, G., Poormohammadi, A. (2020) Sampling and detection of corona viruses in air: A mini review. Sci. Total Environ. 740, 140207. https://doi.org/10.1016/j.s citotenv.2020.140207

Riemenschneider, L., Woo, M.H., Wu, C.Y., Lundgren, D., Wander, J., Lee, J.H., Li, H.W., Heimbuch, B. (2010). Characterization of reaerosolization from impingers in an effort to improve airborne virus sampling. J. Appl. Microbiol. 108, 315-324. https://doi.org/10.1111/j.1365-2672.2009.04 425.x

Rufino de Sousa, N., Shen, L., Silcott, D., Call, C.J., Rothfuchs, A.G. (2020). Operative and technical modifications to the Coriolis ${ }^{\circledR} \mu$ air sampler that improve sample recovery and biosafety during microbiological air sampling. Ann. Work Exposures Health 64, 852-865. https://doi.org/10.109 3/annweh/wxaa053

Seo, G., Lee, G., Kim, M.J., Baek, S.H., Choi, M., Ku, K.B. (2020). Rapid detection of COVID-19 causative virus (SARS-CoV-2) in human nasopharyngeal swab specimens using field-effect transistor-based biosensor. ACS Nano 14, 5135-5142. https://doi.org/10.1021/acsnano.0c02823

Sigaev, G.I., Tolchinsky, A.D., Sigaev, V.I., Soloviev, K.G., Varfolomeev, A.N., Chen, B.T. (2006). Development of a cyclone-based aerosol sampler with recirculating liquid film: Theory and experiment. Aerosol Sci. Technol. 40, 293-308. https://doi.org/10.1080/02786820600596891

Su, L., Du, Q., Tang, M., Qu, Q., Pui, D.Y. (2019). Effects of particle hydrophilicity and morphology on aerosol scavenging in a wet electrostatic scrubber. Aerosol Air Qual. Res. 19, 2331-2340. https://doi.org/10.4209/aaqr.2019.04.0207 
Verreault, D., Moineau, S., Duchaine, C. (2008). Methods for sampling of airborne viruses. Microbiol. Mol. Biol. Rev. 72, 413-444. https://doi.org/10.1128/MMBR.00002-08

Viswanathan, S., Rothamer, D., Zelenyuk, A., Stewart, M., Bell, D. (2017). Experimental investigation of the effect of inlet particle properties on the capture efficiency in an exhaust particulate filter. J. Aerosol Sci. 113, 250-264. https://doi.org/10.1016/j.jaerosci.2017.08.002

Wang, X., Bailey, E.S., Qi, X., Yu, H., Bao, C., Gray, G.C. (2020). Bioaerosol sampling at a live animal market in Kunshan (China): A noninvasive approach for detecting emergent viruses. Open Forum Infect. Dis. 7, ofaa134. https://doi.org/10.1093/ofid/ofaa134

Wilson, N., Corbett, S., Tovey, E. (2020). Airborne transmission of covid-19. BMJ 370, m3206. https://doi.org/10.1136/bmj.m3206

Wong, S.Y., Kwong, R.S., Wu, T.C., Chan, J.W.M., Chu, M.Y., Lee, S.Y. (2020). Risk of nosocomial transmission of coronavirus disease 2019: An experience in a general ward setting in Hong Kong. J. Hosp. Infect. 105, 119-127. https://doi.org/10.1016/j.jhin.2020.03.036

World Health Organization (WHO) (2020a). Modes of transmission of virus causing COVID-19: Implications for IPC precaution recommendations: Scientific brief, 29 March 2020. https://apps.who.int/iris/handle/10665/331616 (accessed 30 December 2020).

World Health Organization (WHO) (2020a). Transmission of SARS-CoV-2: Implications for infection prevention precautions: Scientific brief, 09 July 2020. https://apps.who.int/iris/handle/10665/ 333114 (accessed 30 December 2020).

Yao, M. (2018). Bioaerosol: A bridge and opportunity for many scientific research fields. J. Aerosol Sci. 115, 108-112. https://doi.org/10.1016/j.jaerosci.2017.07.010

Zhang, X., Ji, Z., Yue, Y., Liu, H., Wang, J. (2021). Infection risk assessment of COVID-19 through aerosol transmission: A case study of south China Seafood Market. Environ. Sci. Technol. 55, 4123-4133. https://doi.org/10.1021/acs.est.0c02895

Zhao, B., Su, Y. (2018). Particle size cut performance of aerodynamic cyclone separators: Generalized modeling and characterization by correlating global cyclone dimensions. J. Aerosol Sci. 120, 1-11. https://doi.org/10.1016/j.jaerosci.2018.02.009

Zhao, Y., Aarmink, A.J.A., Wang, W., Fabri, T., PW, G.K., de Jong, M.C. (2014). Airborne virus sampling: Efficiencies of samplers and their detection limits for infectious bursal disease virus (IBDV). Ann. Agric. Environ. Med. 21, 464-471. https://doi.org/10.5604/12321966.1120585

Zheng, Y., Li, J., Chen, H., Zhang, T., Li, X., Wang, M., Yao, M. (2018). Bioaerosol research: Yesterday, today and tomorrow. Chin. Sci. Bull. 63, 878-894. https://doi.org/10.1360/N972018-00121

Zhou, L., Yao, M., Zhang, X., Hu, B., Li, X., Chen, H. (2020). Breath-, air-and surface-borne SARSCoV-2 in hospitals. J. Aerosol Sci. 152, 105693. https://doi.org/10.1016/j.jaerosci.2020.105693

Zuo, Y.Y., Uspal, W.E., Wei, T. (2020). Airborne transmission of COVID-19: Aerosol dispersion, lung deposition, and virus-receptor interactions. ACS Nano 14, 16502-16524. https://doi.org/10.10 21/acsnano.0c08484 